\title{
Rapport annuel 2012
}

Au cours de l'exercice écoulé, la Caisse de secours des médecins suisses a accordé son soutien à 21 médecins tombés malgré eux dans le besoin (ils étaient 22 en 2011) ainsi qu'aux proches et aux descendants de médecins décédés.

Pendant la même période, la Caisse a cessé d'apporter son aide à cinq personnes. Toujours en 2012, quatre nouveaux cas sont venus s'ajouter à la liste des bénéficiaires.

Les produits ont résulté des recettes provenant de dons pour un montant de 115277 CHF (2011: 122157 CHF) ainsi que des intérêts et des dividendes du capital de la fondation, à hauteur de 71412 CHF.

Concernant les charges, les contributions de soutien que nous avons versées ont atteint le montant de 198550 CHF (2011: 200300 CHF). Les dépenses relatives à la gestion de la Caisse de secours et à l'administration de la fortune se sont élevées à 47598 CHF.

La différence entre les recettes provenant de dons ainsi que des intérêts et dividendes du capital et les charges consécutives aux contributions de soutien et aux dépenses administratives a occasionné un résultat négatif de 59981 CHF à la charge du capital de la fondation.

En 2012, la Caisse de secours a reçu un legs important de 533395 CHF sous forme de papiersvaleurs provenant de la succession du Docteur Wadim Rorschach.

Les fondations Dr J. Ambühl, Dr J. Gebhard et Dr J.B. Wutz, qui figuraient jusqu'alors au bilan de manière séparée, ont été dissoutes et le capital a été attribué à la fortune générale de la fondation.

La fortune de la fondation consiste en un mélange d'obligations et d'actions satisfaisant aux prescriptions de la prévoyance professionnelle (LPP). L'apport d'actions en provenance de la succession du Dr Wadim Rorschach a provoqué un surplus de dividendes. Ce déséquilibre sera corrigé au fil du temps.
Le compte d'exploitation, le bilan et la gestion de la fortune sont examinés chaque année par l'autorité de surveillance des fondations et caisses de pension des deux Bâle.

Les membres du conseil de fondation exercent leur activité à titre bénévole.

Les dons individuels inférieurs à $500 \mathrm{CHF}$ forment le pilier traditionnel de notre fondation. Afin de réduire nos frais administratifs au strict minimum, nous renonçons à remercier personnellement les donateurs. Qu'ils veuillent trouver ici l'expression de notre vive gratitude.

Les comptes annuels de la Caisse de secours ont été révisés par la fiduciaire Axt Treuhand qui les a trouvés conformes aux règles. Nous la remercions cordialement de son travail. Le lecteur intéressé trouvera des informations détaillées sur les comptes annuels dans le bilan et le compte d'exploitation publiés ci-après.

Nous remercions chaleureusement tous les membres de la Commission pour le travail qu'ils ont accompli à titre bénévole durant l'exercice écoulé. Nous adressons également nos sincères remerciements aux Messieurs J. Kobler et W. Mahrer de la Banque La Roche 1787 pour la tenue de la comptabilité et la gestion de la fortune.

La Commission de fondation de la Caisse de secours des médecins suisses:

\section{Dr U. Leibundgut, président}

DrS. Stöhr

Dr U. Lüscher, trésorier

Compte postal de la Caisse de secours des médecins suisses: 40-644-3 Bâle
Dr U. Leibundgut Lange Gasse 78 CH-4052 Bâle

\footnotetext{
dendes. Ce déséquilibre sera corrigé au fil du temps.
} 


\section{Comptes annuels 2012 (en francs)}

Bilan au 31 décembre 2012

Comptes Banque La Roche

Compte postal

Débiteur impôt anticipé

Titres

Compte de régularisation des passifs

Provision sur titres

Fortune au 31 décembre 2012

Héritage

Liquidation Fonds (Ambühl / Genhard / Wutz)

Caisse de secours au $1^{\text {er janvier }} 2012$

Changement de l'exercice

Compte d'exploitation pour 2012

Dons

Revenu sur capital

Ajustement provision sur titres

Pertes sur titres (réal.)

Ajustement change

Contributions

Frais de banque et d'administration

Perte de l'exercice

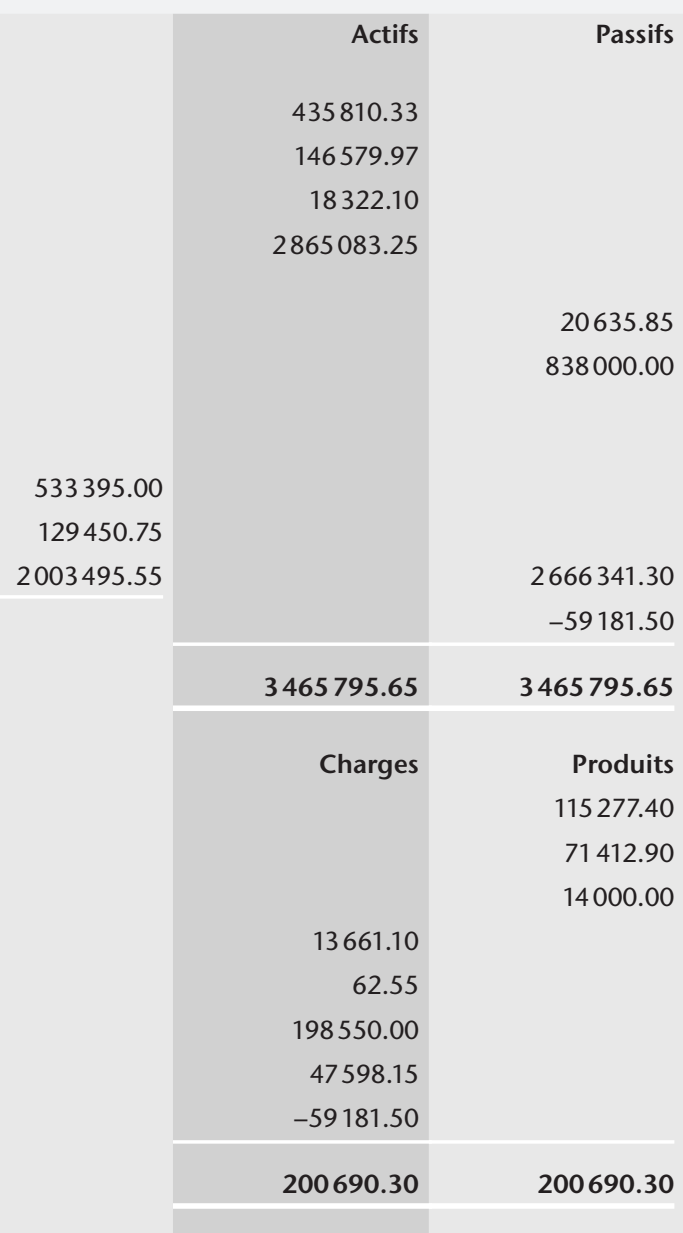

